Chapter 4

\title{
Nanotechnologies Applied in Biomedical Vaccines
}

\author{
Yuan-Chuan Chen, Hwei-Fang Cheng, \\ Yi-Chen Yang and Ming-Kung Yeh \\ Additional information is available at the end of the chapter \\ http://dx.doi.org/10.5772/63453
}

\begin{abstract}
Vaccination, one of the most effective strategies to prevent infectious diseases, is the administration of antigenic materials to stimulate an individual's immune system to develop adaptive immunity to a specific pathogen. Though it is so advantageous for diseases control and prevention, vaccines still have some limitations. Nanotechnology is an approach to prepare a novel biomedicine vaccine with the vaccine consumption and side effects significantly decreased. Regulation is the most important criterion for the development of nanovaccines. All marketing products have to meet the requirement of regulation. The fast-track designation potentially aids in the development and expedites the review of nanovaccines that show promises in an unmet medical need. Here, somesuccessful nanovaccine productsareintroduced-Inflexal ${ }^{\circledR} \mathrm{V}$, Epaxal ${ }^{\circledR}$, Gardasil ${ }^{\mathrm{TM}}$, and Cervarix ${ }^{\mathrm{TM}}$ have been widely used for the clinical applications, which are delivered either in the form of virosomes or virus-like particles. Vaccines based on nanotechnology may overcome their original disadvantages and lead to the development of painless, safer, and more effective products.
\end{abstract}

Keywords: biomedicine, clinical application, fast track, nanotechnology, vaccine

\section{Introduction}

The terms vaccine and vaccination, derived from Variolae vaccinae (smallpox of the cow), were devised by Edward Jenner to denote cowpox. Edward Jenner was an English doctor and scientist who was the pioneer of smallpox vaccine, the first vaccine in the world. In 1796, Jenner took pus from the hand of a milkmaid with cowpox and scratched it into the arm of an 8-yearold boy. Six weeks later, he inoculated the boy with smallpox, afterwards observing that he did not catch smallpox and no diseases followed [1,2]. The boy was further challenged with 


\begin{tabular}{|c|c|c|}
\hline Type & Definition & Example \\
\hline Inactivated & $\begin{array}{l}\text { Virulent microbes are previously } \\
\text { destroyed with chemicals, heat, } \\
\text { radiation, or antibiotics }\end{array}$ & $\begin{array}{l}\text { Influenza vaccine, whole cell pertussis } \\
\text { vaccine, cholera vaccine, hepatitis A vaccine, } \\
\text { rabies vaccine, plague vaccine, inactivated } \\
\text { polio vaccine (IPV) }\end{array}$ \\
\hline Attenuated & $\begin{array}{l}\text { Live microbes are cultivated under } \\
\text { conditions to disable their virulence, } \\
\text { or closely related but less dangerous } \\
\text { microbes are used to induce broad } \\
\text { immune responses }\end{array}$ & $\begin{array}{l}\text { Yellow fever vaccine, shingles vaccine, } \\
\text { measles, mumps and rubella (MMR) vaccine, }{ }^{(1)} \text { typhoid } \\
\text { (Ty21a) vaccine, BCG vaccine, rotavirus vaccine, } \\
\text { live attenuated influenza vaccine (LAIV), } \\
\text { oral polio vaccine (OPV) }\end{array}$ \\
\hline Toxoid & $\begin{array}{l}\text { Vaccines are made from inactivated } \\
\text { toxic compounds that cause illness } \\
\text { rather than microbes }\end{array}$ & Tetanus toxoid, diphtheria toxoid \\
\hline \multirow[t]{3}{*}{ Subunit } & $\begin{array}{l}\text { Protein-based vaccine: A fragment of } \\
\text { protein creates an immune response, } \\
\text { rather than introducing inactivated } \\
\text { or attenuated microbes to an immune } \\
\text { system. }\end{array}$ & $\begin{array}{l}{ }^{(2)} \text { Hepatitis B vaccine, }{ }^{(2)} \text { human } \\
\text { papillomavirus (HPV) vaccine }\end{array}$ \\
\hline & $\begin{array}{l}\text { Polysaccharide vaccine: A } \\
\text { capsular polysaccharide antigen } \\
\text { is covalently attached to a } \\
\text { carrier protein and creates an immune } \\
\text { response. }\end{array}$ & $\begin{array}{l}{ }^{(1)} \text { typhoid (Vi) vaccine, } \\
{ }^{(3)} \text { pneumococcus vaccine, } \\
{ }^{(4)} \text { meningococcus vaccine }\end{array}$ \\
\hline & $\begin{array}{l}\text { Conjugate vaccine: Certain } \\
\text { pathogens have poorly immunogenic } \\
\text { polysaccharide outer coats. The } \\
\text { immune system recognizes the } \\
\text { polysaccharide as if it were a } \\
\text { protein antigen by linking } \\
\text { these outer coats to proteins }\end{array}$ & $\begin{array}{l}\text { Haemophilus influenzae } \\
\text { type b (Hib) vaccine, }{ }^{(3)} \text { pneumococcus } \\
\text { vaccine, }{ }^{(4)} \text { meningococcus vaccine }\end{array}$ \\
\hline
\end{tabular}

Table 1. The major types of vaccines in the clinical application represent different strategies used to reduce the risk of illness, while retaining the ability to induce beneficial immune responses.

various materials and again showed no signs and symptoms. In 1798, Jenner extended his studies and reported that his vaccine was safe and could be transferred from arm-to-arm to reduce dependence on uncertain supplies from infected cows [3]. In the 1880s, the second generation of vaccines was introduced by Louis Pasteur who developed vaccines for chicken cholera and anthrax [4]. In 1881, to honor Jenner, Louis Pasteur proposed that the terms should be extended to cover the new protective inoculations then being developed [4]. From the late nineteenth century, vaccines were considered a matter of national prestige, and vaccination is a compulsory policy in many countries [5]. In the twentieth century, several successful vaccines were introduced such as those against diphtheria, measles, mumps, and 
rubella. During this period, the development of the polio vaccine in the 1950s and the eradication of smallpox around 1960s-1970s are great achievements. As vaccines had already become more common, many people began taking them for granted.

A vaccine, an antigenic material, is a biological preparation that provides adaptive immunity to a specific disease. The agent triggers immune responses to recognize the disease-causing agent to destroy and remember it, so that the immune system can immediately recognize and destroy the foreign agent once it invades into the body later. Inactivated microbes live attenuated microbes, toxoids, subunits, or conjugates have been manufactured as vaccines and employed to stimulate adaptive immune responses (Table 1). A monovalent (univalent) vaccine is designed to immunize against a single antigen or microorganism such as measles vaccine, while a multivalent (polyvalent) vaccine is designed to immunize against more than one strain of the same microorganism or against more than one microorganism such as oral polio vaccine (OPV), three in one (e.g., diphtheria-tetanus-pertussis, DTP) vaccine, etc. Heterologous vaccines are pathogens of other animals that do not cause diseases or only cause mild diseases in the organism being treated, such as the cowpox vaccine and the Bacillus Calmette-Guérin (BCG) vaccine.

(1) Two types of typhoid vaccines are available: typhoid (Ty21a) vaccine (a live, attenuated vaccine given by mouth) and typhoid (Vi) vaccine (a subunit vaccine given by injection). (2) Current hepatitis B vaccine and human papillomavirus (HPV) vaccine may be produced by recombinant DNA technology; therefore, they are sometimes called recombinant vaccines. (3) Two types of pneumococcus vaccines given by injection are available: pneumococcal polysaccharide vaccine (PPSV) and pneumococcal conjugate vaccine (PCV). (4) Two types of meningococcus vaccines given by injection are available: meningococcal polysaccharide vaccine (MPSV) and meningococcal conjugate vaccine (MCV).

It is well-known that prevention is better than cure for diseases control. Vaccination is the best approach to prevent infectious diseases ahead of the incidence of diseases. Vaccination can prevent or ameliorate morbidity from infection and result in herd immunity when a high percentage of population has been vaccinated. For the past centuries, some diseases are globally eradicated such as smallpox; some diseases are significantly controlled in much of the world such as polio, measles, and tetanus through vaccines distribution. The effectiveness of vaccination has been widely studied and verified such as the influenza vaccine [6], HPV (Human papillomavirus) vaccine [7], and the shingles (chicken pox) vaccine [8], etc. In 2012, the World Health Organization (WHO) reports that licensed vaccines are currently available to contribute to the prevention and control of 25 infections. From the discovery of the related pathogens, 25 infection have been effectively prevented using licensed vaccines; however, vaccines remain elusive for many important diseases, such as the infections of Zika viruses, cytomegaloviruses (CMV), hepatitis C viruses (HCV), Human immunodeficiency viruses (HIV), severe acute respiratory syndrome (SARS) viruses, Ebola viruses, respiratory syncytial viruses (RSV), and malaria parasites Plasmodium, etc. (Figure 1). A vaccine is not only conventionally prophylactic, hinting to prevent or alleviate the effects of a future infection, but also may be therapeutic, vaccines against cancers are being investigated in recent research such 
as the HPV vaccine for cervical cancer, the BCG vaccine for bladder cancer [9] and colorectal cancer [10].

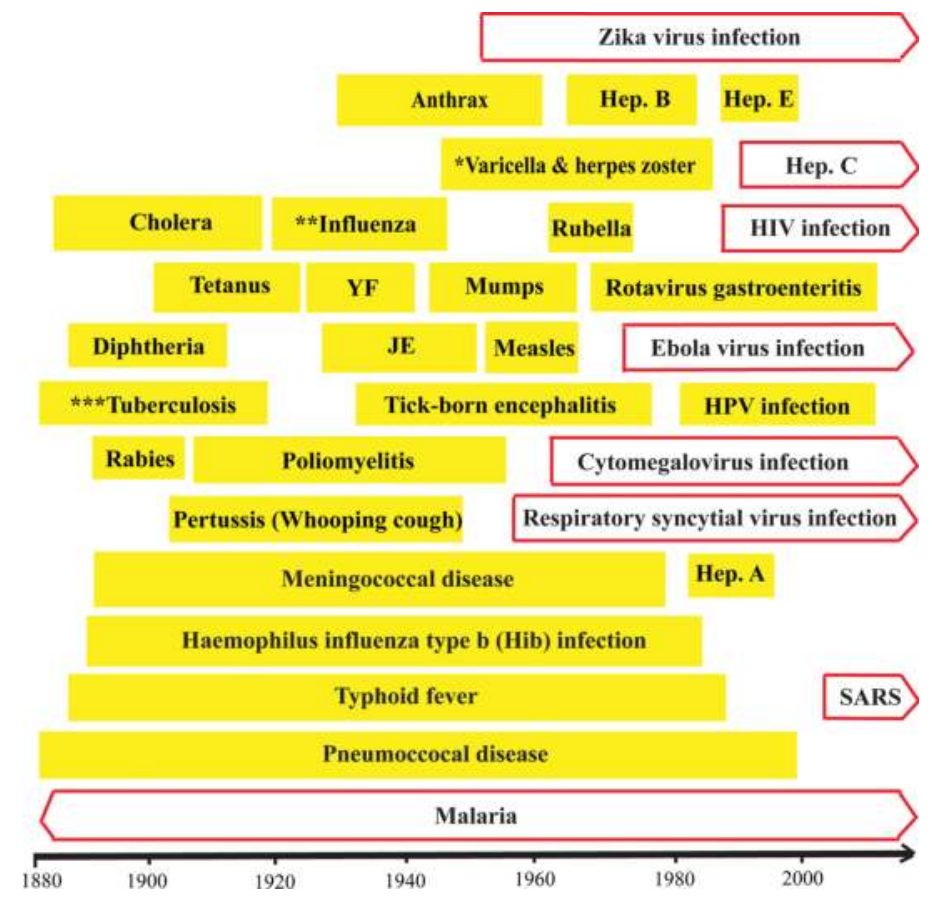

Figure 1. Time line for the development of vaccines: 25 infections ( yellow column) have been reported by WHO being prevented using licensed vaccines. However, some infections under research have no effective vaccines available yet ( $\square$ blank arrow column). *Varicella and herpes zoster: Varicella (chicken pox) and herpes zoster (shingles) are diseases caused by varicella-zoster viruses (VZV) (one of the eight herpesviruses known to infect humans and vertebrates). ${ }^{* *}$ Influenza: A new version of the influenza vaccine is usually developed twice a year, because influenza viruses mutate rapidly. ${ }^{* * *}$ Tuberculosis (TB): The Bacillus Calmette-Guérin (BCG) vaccine was originally developed from Mycobacterium bovis found in cows; therefore, its efficacy against pulmonary TB appears to be variable in humans. Abbreviations: Hepatitis (Hep.); Human papillomavirus (HPV); Human immunodeficiency virus (HIV); Japanese encephalitis (JE); Severe acute respiratory syndrome (SARS); Yellow fever (YF).

Vaccines have not only successfully reduced the incidence rate of many devastating diseases to be always low but also prevented many infectious diseases from persistently bursting out. At present, only few people have ever experienced the detrimental effects of many illnesses. The two main advantages of vaccination are noted as follows:

\section{Prevention in advance}

Vaccination can result in lower morbidity and mortality rate as well as faster recovery even though a partial, late, or weak immunity may only alleviate an infection. The early protective efficacy of vaccination is primarily conferred by the induction of antigenspecific antibodies. Vaccination can trigger adaptive immunity before contacting with the 
pathogen. Should a pathogen infect the body, there is no illness in that the immune responses stimulated by vaccines continuously guard against the disease. The quality of such antibody responses (avidity: a measure of the overall strength of an antigen-antibody complex) has been identified as a determining factor of vaccines efficacy. However, there is more to antibody-mediated protection than the peak of vaccine-induced antibody titers. Vaccination stimulates both humoral and cell-mediated immunity of one's own throughout the body. This kind of defensive response is usually safer, more effective and with fewer side effects (e.g., inflammation, muscle aches, allergy, etc.) due to the active, acquired and specific immunity is established. Through vaccination, humans can eliminate or alleviate the attack of pathogens beforehand to avoid the suffering of diseases.

2. Immunity for long term

Vaccination stimulates adaptive immunity to create immunological memory that can maintain in the body for a long period of time. Therefore, people will be protected from the attack of diseases as long as these specific memory cells are still in the body. The disease control or elimination requires the induction of protective immunity in a sufficient proportion of the population. This is best achieved by vaccination programs capable of inducing the long term protection (adaptive immunity) that contrasts to the quick but short-lasting immune responses (innate immunity). Long-term immunity is conferred by the maintenance of antigen-specific immune effectors and/or by the induction of immune memory cells that may be efficiently and rapidly reactivated upon subsequent microbial exposure.

In spite of vaccines' advantages for diseases control and prevention, three major limitations related to vaccines are noted as follows:

1. Complex vaccination schedules

The potency of vaccines may not be high enough in receiving immunization only once. Though the body develops antibodies, protection sometimes is not adequate due to the development of immunity might be too slow to be effective in time. Furthermore, the antibodies might not disable the pathogen completely, or there might be multiple strains of the pathogen, not all of which are equally susceptible to the immune responses. In order to provide the best protection, additional "booster" shots are often required to achieve full immunity. Vaccine schedules are usually regulated by the competent authority or physician groups to achieve maximum effectiveness. A vaccination schedule is a series of vaccinations, including the timing of all doses, which may be either recommended or compulsory. Many vaccines require multiple doses for maximum effectiveness, either to produce sufficient initial immune responses or to boost responses that fade over time. Over the past two decades, the recommended vaccination schedule has grown rapidly and become more complex as many new vaccines have been developed.

2. Strict requirements for storage and shipment

Vaccines are temperature sensitive and their storage and shipment are usually restricted to cold temperature [11]. Potency can be negatively affected by exposing to extended 
or multiple temperature excursions (out-of-range temperatures). It is essential to have a label on the storage unit to indicate that vaccines should be stored in the freezer $(-20$ or $-80^{\circ} \mathrm{C}$ ) or in the refrigerator $\left(2-8^{\circ} \mathrm{C}\right)$. Correct cold-chain (a temperature-controlled supply chain) procedures must be followed during production, warehousing, and transportation, that is, vaccines have to be stored properly from the time that they are manufactured, distributed until administered to the people. Most live, attenuated vaccines tolerate freezing temperatures, but deteriorate rapidly after being removed from cold temperature. Inactivated vaccines will be damaged by exposing to temperature fluctuations. Coldchain failure has to be evaluated in addition to routine tests such as potency, sterility, toxicity, safety, chemical, visual, and pyrogen test.

3. Restricted routes of administration

Most vaccine products are administered by injection such as intramuscular (IM), subcutaneous (SC), and intradermal (ID) injection, though some can be given by oral administration or intranasal spray. IM injection administers the vaccine into the muscle mass. Vaccines containing adjuvants should be injected into muscle to reduce adverse local effects. SC injection administers the vaccine into the subcutaneous layer above the muscle and below the skin. ID injection administers the vaccine in the topmost (dermis) layer of the skin. BCG is the known vaccine with ID injection; this route reduces the risk of neurovascular injury. The professional person's assistance is usually required for the injection administration. Oral administration vaccines (e.g., OPV and rotavirus vaccine) make immunization easier by self-administration and eliminating the need of an injector. The intranasal spray vaccine (e.g., influenza vaccine live) also offers a needle-free approach through the nasal mucosa. Percutaneous administration, such as a multiple puncture device (4-5 cm length, with 9 short needles) used for BCG vaccination in Japan, indicates that any medical procedure is done via needle puncture of the skin, rather than using an "open" approach where inner organs or tissues are exposed.

In the new era of subunit vaccines, to against both infectious diseases and cancers effectively, subunits made of proteins, peptides, DNA antigens, or potent adjuvants may provide a good choice to enhance immunogenicity. Subunit vaccines are not generally immunogenic in humans because of size, degradation, destruction, nonspecific targeting, lack of crosspresentation, and others [12]. This reveals that it is necessary to develop a new approach to package and present antigens to the immune system. Nanomedicine is an area of research that combines nanotechnology and medicine and provides new hopes for therapeutic strategies and diseases control.

Advances in nanotechnology have shown to be beneficial for therapeutic strategies such as drug discovery, drug delivery, and gene/protein delivery. Through nanotechnology, drug consumption and side effects can be significantly decreased by locating the active agent at the desired site. Nanotechnology is the study and application of extremely small things and capable of being used in many science fields, such as biology, chemistry, physics, materials science, and engineering. It is referred to the particular technology with a goal to precisely manipulate atoms and molecules for fabrication of nanoscale products, which size below 100 $\mathrm{nm}$ (or sometimes up to 200-300 nm) [12] (Figure 2). Advances in nanotechnology in biome- 
dicines have proved to be beneficial in vaccines development. The efficacy of vaccines can be improved and side effects also can be significantly decreased. For the past decades, a nanovaccine has become a novel approach for vaccination.

Adjuvants may be added to vaccines to modify the immune response by boosting it to give more antibodies and longer-lasting protection. Adjuvants have been an important component of vaccine formulations since aluminum salt (Alum) was approved in the 1920s [13] and adjuvant system 04 (AS04) was approved in 2009 in the United States, respectively. AS04 is a trade name for combination of adjuvants used in vaccine products by GlaxoSmithKline (GSK), which consists of aluminum hydroxide $\left(\mathrm{Al}(\mathrm{OH})_{3}\right)$ and 3-O-desacyl-4-monophosphoryl lipid A (MPLA). The toll-like receptor (TLR) agonist and MPLA are being intensively investigated as part of a new class of biological adjuvants [14]. In Europe, a few other adjuvants based on oil in water emulsions such as adjuvant system 03 (AS03) and liposomes (made of lipid vesicles) have been approved for using as adjuvants in humans by the European Medicines Agency (EMA) since the 1990s [15]. AS03 is a trade name for a squalene-based immunologic adjuvant used in vaccine products by GSK, which contains squalene, DL- $\alpha$-tocopherol and polysorbate 80 . For a great need for potent adjuvants in the vaccine clinical applications, we believe that nanotechnology could be well positioned to take on such a challenge. Consequently, we focus on the vaccine products based on nanotechnology. In this chapter, we review the regulation related to the development of nanovaccines, already marketed products based on nanotechnology and the perspective of developing highly effective, stable, and economic vaccines using micro-/nanoparticles.

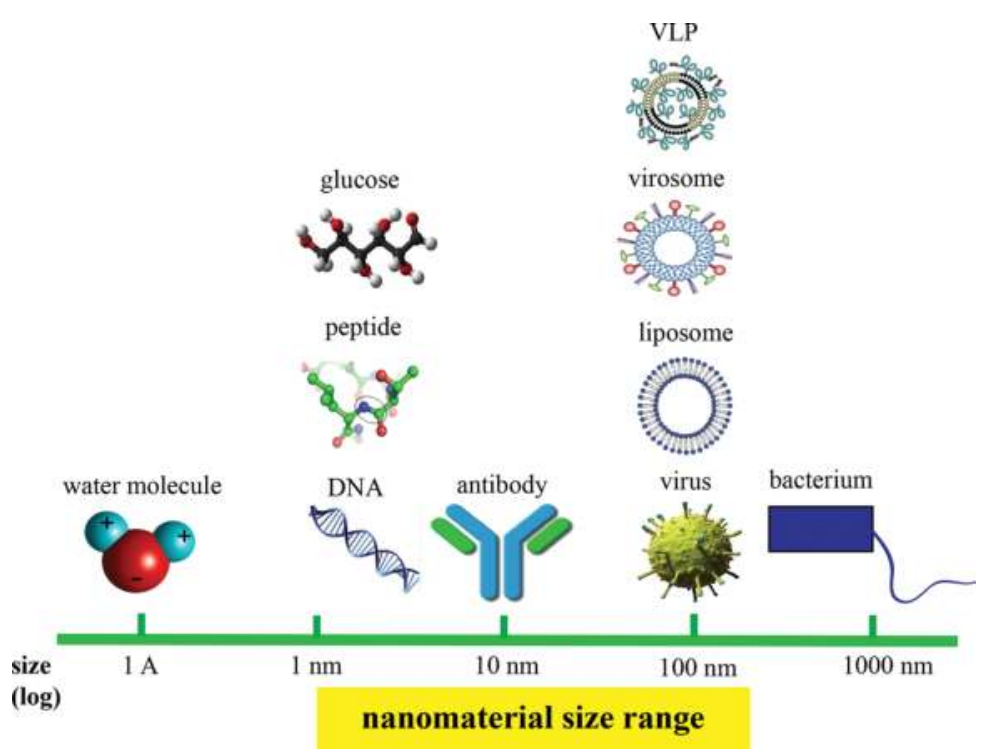

Figure 2. Size comparison of biological substances: The size range of nanomaterials used in nanotechnology relative to the size of various biological system. Abbreviation: virus-like particle (VLP). 


\section{Regulation}

In accordance with the guidelines of WHO, US Food and Drug Administration (FDA) and EMA, vaccines belong to a heterogeneous class of agent; each has suitable, individual, and specific standards of evaluation. The major quality concerns in a vaccine human clinical trial are as follows:

1. Theoretical values and expected values of the trial: In preclinical studies stage, potency, and immunogenicity should be tested in addition to toxicity and safety. A suitable animal challenging model should be established; otherwise, an alternative method must be proposed. The contribution and clinical significance of vaccine should be explained, and a declaration of interest is usually needed.

2. Protection of the testers: The right and interest of testers should be emphasized either in the placebo control group (e.g., use insert placebos) or the active control group (e.g., use already licensed vaccines).

3. Selection of testing population group: Random health volunteers are tested in phase I, but the representation of testers' population should be considered in phase II and III. The related factors include testers' population, social economics, and the epidemiology of target disease, etc.

4. Condition of inclusion and exclusion for testing population group: Tester should be in good health condition and meet the criteria of examination, some people would be excluded such as allergy, cardiovascular disease, immunodeficiency, and neuropathy sicks.

5. Dosage, administration route, and schedule: Dose-response data should be achieved in the dose-finding study. The schedule of vaccination depends on the traits of antigens, the characteristics of testing population, and the pharmacokinetics induced by vaccines. Tolerance, safety, dosage, and administration routes should be evaluated in phase I. The optimal dose and schedule of vaccination should be defined in phase II and would be a reference in phase III.

6. Safety evaluation of testers: The adverse events should be recorded and evaluated in all phases of the clinical trial. Post-marketing surveillance for side effects in phase IV is usually required.

7. Limitation of concomitant administration: The interaction between drugs and vaccines is rare, but it is necessary to record the consequence while concomitant administering them.

8. Efficacy and effectiveness of vaccines: Vaccine efficacy is the measure of the reduced disease incidence after vaccination, compared with the unvaccinated group. Vaccine effectiveness is the measure of vaccine induced protection (not population related protection), which is influenced with many factors such as the protection coverage of vaccination, the immunological state of testers, and the epidemiology of target disease. 
9. Lot-to-lot consistency study: It is necessary to evaluate the consistency of vaccine production and performance by comparing the different lots of vaccine products by the predefined standard (usually various parameters in immunology).

Vaccines, as all medicinal products regulated by national regulatory authorities (NRA) and national control laboratories (NCL), undergo a rigorous review of laboratory and clinical data to ensure their safety, efficacy, purity, and potency. In the stages of discovery and development of vaccines, the manufacturer has to comply with the related regulation, including nonclinical work, consultation, preclinical trials, and human clinical trials. Furthermore, vaccines approved for marketing may also be required to perform additional studies to give further evaluation and often address specific questions such as safety, efficacy or possible side effects and contraindications for post-marketing surveillance (Figure 3).

Lot release is an independent assessment of each lot of a licensed vaccine or biologic prior to it is released onto the market. General practices for lot release involve the review of manufac-

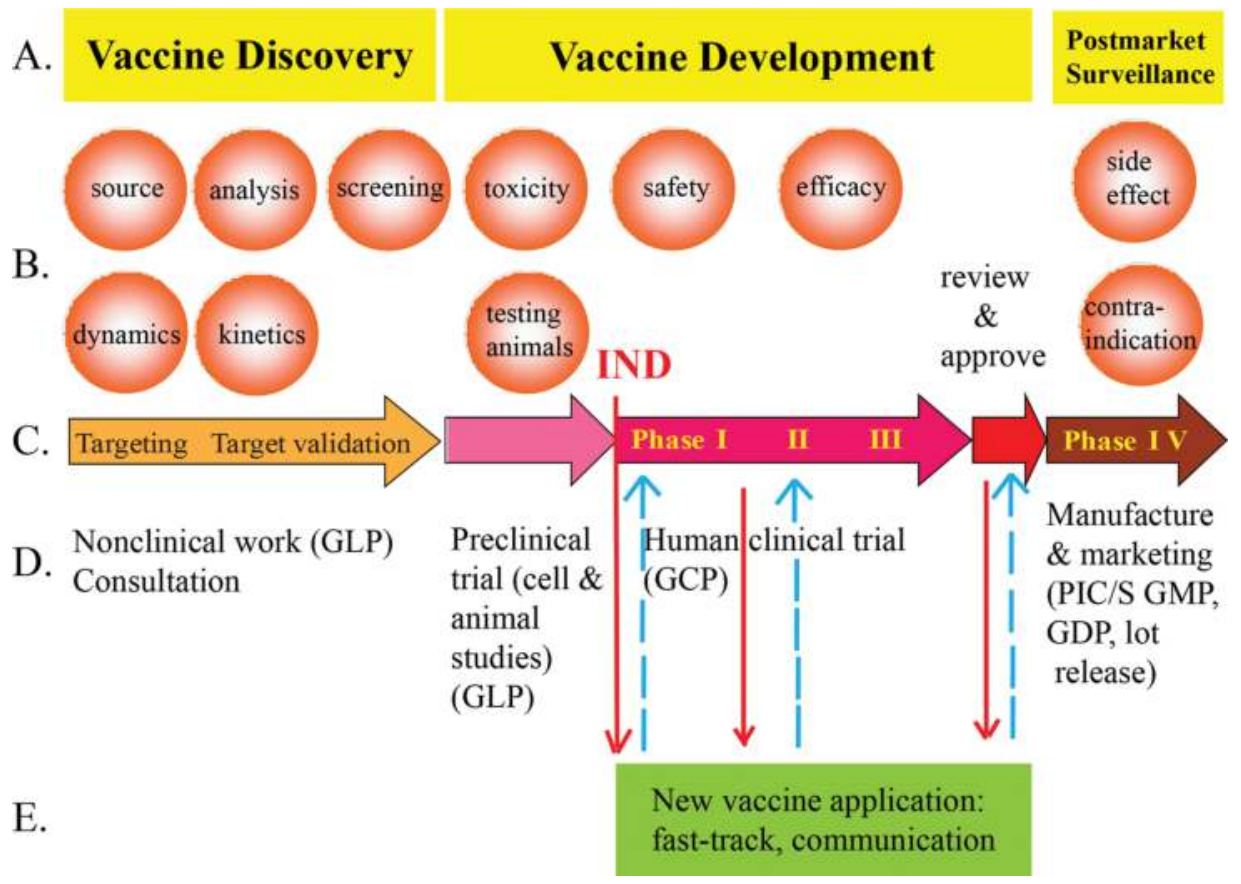

Figure 3. Frame work of vaccine research and development: The research, development, surveillance, and application of vaccines involve several practices performance and regulation requirements. (A) Stage: Vaccines discovery, development, and postmarked surveillance are three stages for the application of new vaccines. (B) Studies: There is much research work to do at every stage. (C) Phase: Each stage can be divided into different phases. (D) Regulation: Various regulations or guidelines are required for every stage. (E) Special procedure: fast track and communication. Abbreviations: Good Laboratory Practice (GLP); Good Clinical Practice (GCP); Good Distribution Practice (GDP); Investigational New Drug (IND), Pharmaceutical Inspection Convention and Pharmaceutical Inspection Co-operation Scheme/ Good Manufacturing Practice (PIC/S GMP). 
turers' production data and quality control test results by NRA and NCL. These practices can be performed by an agency or a contracted laboratory for the NRA. It may be supplemented by other documents such as the release certificate from the responsible NRA/NCL and in some circumstances, by tests in the other laboratory which is independent of the manufacturers' quality control testing.

Fast-track procedures can be implemented under special circumstances, such as disease outbreaks or an acute shortage in global vaccine supply. It is a process designed to facilitate the development and expedite the review of drugs that show promise in treating a serious or life-threatening disease and address an unmet medical need as follows:

1. Serious condition: To determine if the drug will have an impact on such factors as survival, day-to-day functioning or the disease will progress more serious if left untreated such as acquired immune deficiency syndrome (AIDS), Alzheimer's disease, heart failure, cancer, epilepsy, depression, and diabetes.

2. Unmet medical need: The drug must be developed to treat or prevent a disease that does not have a current therapy. If there are available therapies, a fast-track drug must show some advantage over available therapy such as showing of superior effectiveness, avoidance of serious side effects, improvement of the diagnosis, decrease of clinically significant toxicity, and the need of emerging or anticipated public health.

The purpose of a fast-track designation is to get important new drugs to the patient earlier through the consultation system. Once a drug receives fast-track designation, early and frequent communication between the NRA and a drug manufacturer is encouraged throughout the entire development and review process of drugs. The communication ensures that all questions and issues can be solved quickly, often leading to earlier drug approval and access by patients. For example, US FDA is able to offer accelerated approval or priority review if the requisite criteria are met. Priority review rapids the review process for a new drug from 6 to 10 months even can have US FDA make a final decision within 60 days.

In the application of nanoparticles as vaccines, some obstacles need to be overcome such as stability during manufacturing and storage, sterilization by nonthermal methods, and reproducibility of formulation during large-scale production [16]. Reproducibility of formulation during manufacturing is one of the major hurdles [17]; for instance, size-dependent immunogenicity has been reported [18] and it is observed that nanomaterials may change toxicity only by changing size and shape but not composition [19]. If the nanoparticles are cleared slowly over a long period of time, they may induce toxic effect. In addition, large counterparts may accumulate in vital organs to cause toxicity, even though small nanoparticles can be removed quickly from the body. Because of the toxic concerns of nanomaterials, the potential effects on the human body, public health, and environment pollution should be taken into account. It is necessary to evaluate the effects on DNA damage, gene expression, cell death, and growth in the body when different types of cells are exposed to nanoparticles. The safety surveillance and quality control of nanovaccines are as central as the evaluation of their efficacy. Regulatory policies are based mainly on the purity and safety of the vaccine. For 
risk management, some special regulations or guidelines for nanotechnology application in vaccines are as follows.

1. The approach to nanotechnology products
A. Product-focused and science-based regulatory policy
B. Attention to nanomaterials being incorporated into standing procedures
C. In legal standards for different product-classes

2. The regulation of pharmacopoeia (European Pharmacopeia 7.0 and 8.0; EP7.0, 8.0)

A. General provisions of products: The production method is validated to demonstrate that the product would comply with the test for abnormal toxicity for immunosera and vaccine for human use. Additionally, this method has to yield consistent vaccines comparable with the vaccine of proven clinical efficacy and safety in humans.

B. Preparation of nanoparticles (virosomes): Inactivated influenza virions are solubilized using a suitable detergent and purified by high-speed centrifugation to obtain supernatants containing mainly influenza antigens. After the addition of suitable phospholipids, virosomes are formed by removal of the detergent either by adsorption chromatography or another suitable technique.

C. Tests of final products: residual infectious virus, $\mathrm{pH}$, phospholipids, ratio of hemagglutinin to phospholipids, antimicrobial preservative, free formaldehyde, ovalbumin, total protein, sterility, virosome size, and bacterial endotoxin

3. Preclinical efficacy and toxicity testing of nanoparticles

A. Physicochemical characterization: size measurement, topology, molecular weight, aggregation, purity, chemical composition, surface characteristics, functionality, zeta potential, stability, and solubility test

B. In vitro characterization: sterility, targeting, drug release, in vitro immunology, and toxicity test

C. In vivo characterization: efficacy and repeated-dose toxicity test

\section{Products in the clinical application using micro/nanoparticles}

Inflexal ${ }^{\circledR} \mathrm{V}$, Epaxal ${ }^{\circ}$, Gardasil $^{\mathrm{TM}}$, and Cervarix ${ }^{\mathrm{TM}}$, marketed in many countries for over 10 years, are four common vaccine products based on micro/nanotechnologies. They use nanoparticles-virosomes (special liposomes) or virus-like particles (VLPs) as a delivery and adjuvant system, and all should be administered by IM and stored at $2-8^{\circ} \mathrm{C}$ (Table 2). 


\begin{tabular}{llll}
\hline Product & Inflexal ${ }^{\circledR}$ V & Epaxal ${ }^{\circledR}$ & ${ }^{(1)}$ Gardasil $^{\mathrm{TM}}$ and $^{(2)}$ Cervarix $^{\mathrm{TM}}$ \\
\hline Indication & Influenza & Hepatitis A & Cervical cancer \\
& a mixture of three & a vaccine adjuvanted & ${ }^{(1)}$ Aluminum adjuvant \\
& monovalent virosome & with IRIV & composed of AAHS \\
& pools & & ${ }^{(2)} \mathrm{ASO}$ adjuvant composed \\
& & of Al $(\mathrm{OH})_{3}$ and MPLA \\
Nanoparticle & Virosome & Virosome & VLP \\
Size in diameter & $150 \mathrm{~nm}(100-300 \mathrm{~nm})$ & $150 \mathrm{~nm}(100-300 \mathrm{~nm})$ & $20-60 \mathrm{~nm}$ \\
\hline
\end{tabular}

Abbreviations: adjuvant system 04 (AS04); amorphous aluminum hydroxyphosphate sulfate (AAHS); aluminum hydroxide $\left(\mathrm{Al}(\mathrm{OH})_{3}\right)$; immunopotentiating reconstituted influenza virosomes (IRIV); 3-O-desacyl-4-monophosphoryl lipid A (MPLA); virus-like particle (VLP).

Table 2. Comparison of different vaccine products based on nanotechnology.

A virosome is a kind of vaccine delivery tool with a typical size in diameter about $150 \mathrm{~nm}$. It consists of a phospholipid membrane vesicle incorporating viral-derived proteins and tends to form a pure fusion-active vesicle. Virosomes are only able to fuse with target cells without replicating inside their hosts. In contrast to liposomes, virosomes contain functional viral envelope glycoproteins: influenza virus hemagglutinin (HA) and neuraminidase (NA) intercalated in the phospholipid bilayer membrane. Virosomes represent an innovative, extensively applicable adjuvant and carrier system and are used in areas beyond conventional vaccines.

Virus-like particles (VLPs), derived from viruses and having no genetic materials, are selfassembling nanoparticles (20-60 $\mathrm{nm}$ in diameter) that expose multiple epitopes on their surface and resemble native virions [20,21]. VLPs can differentiate themselves from soluble recombinant antigens, and their structure is capable of inducing stronger immune responses. They also can express one or several viral structural proteins in a recombinant heterologous system [22], for example, the recombinant baculovirus system results in the expression of massive and various virus proteins. It is possible to engineer vaccines with multiple viral epitopes to elicit protective immunity due to the availability of viral three-dimensional structures [23]. VLPs co-administered with oil, mutant E. coli heat-labile toxins in calves [24] and with bluetongue viruses in sheep [25] have provided protection against the related viruses. The immunogenicity of VLPs may be induced because of their interaction with dendritic cells (DCs) and has been a useful approach for immunity against viruses in animals [16].

\subsection{Inflexal ${ }^{\circledR} \mathrm{V}$}

Influenza is an important respiratory infection of humans, responsible for many deaths worldwide every year. The influenza viruses are genetic variable due to continuous and gradual mutation as well as rearranged genome segments between viruses [26]. Frequent mutations of the surface glycoproteins HA and NA result in the gradual evolution of viral strains (antigenic drift). Consequently, it is required to develop a version of new influenza 
vaccine almost every year in that infectious viruses can no longer be neutralized effectively by the antibodies raised against previously circulating strains.

Inflexal ${ }^{\circledR} \mathrm{V}$, an influenza vaccine, is composed of a mixture of three monovalent virosome pools, each formed with specific glycoproteins HA and NA of one influenza virus strain. The vaccine is completely biodegradable and contains no thiomersal, formaldehyde, and very low levels of ovalbumin. Virosomes, reconstituted by nanotechnology, can deliver influenza antigens to stimulate a strong immune response; thus, this product is highly efficacious by mimicking natural viral infection [22]. The product has been approved by many countries and is the only adjuvanted influenza vaccine licensed for all age groups.

\subsection{Epaxal ${ }^{\circledR}$}

Hepatitis A is an acute infectious disease of the liver caused by the hepatitis A virus (HAV), which symptoms include nausea, vomiting, diarrhea, jaundice, fever, and abdominal pain. Around $10-15 \%$ of people experience a recurrence of symptoms during the six months after the initial infection. Acute liver failure may rarely occur with this being more common in the elderly.

Epaxal®, a hepatitis A vaccine, is adjuvanted with immunopotentiating reconstituted influenza virosomes (IRIV). This vaccine is based on formalin inactivated HAV adsorbed to the surface of virosomes that replace the adjuvant-aluminum hydroxide with phospholipids. A single injection of virosomal HAV vaccine is well tolerated and highly immunogenic, with $88-97 \%$ of seroprotection 2 weeks after the first dose (reinforcing immunization: one dose at 6-12 months after the first dose).

\subsection{Gardasil ${ }^{\mathrm{TM}}$ and Cervarix ${ }^{\mathrm{TM}}$}

HPV is a sexually transmitted infection and results in serious illness including genital warts and cervical cancers. Cervical cancer is the second most common cause of cancer deaths in females worldwide, only next to breast cancer [27]. HPV causes many cervical cancer cases every year, of all the cases, type 16 and 18 are responsible for about $70 \%$.

Gardasil $^{\mathrm{TM}}$ and Cervarix ${ }^{\mathrm{TM}}$ are subunit vaccines containing VLPs assembled from the major capsid protein (L1 protein) of HPV type 6, 11, 16, and 18 (Gardasil ${ }^{\mathrm{TM}}$ ) and type 16 and18 $\left(\right.$ Cervarix $\left.^{\mathrm{TM}}\right)$ [21]. Vaccination with these two products has been shown to protect women against a high proportion of precursor lesions of cervical cancer caused by HPV.

Gardasil $^{\mathrm{TM}}$ is a sterile liquid suspension prepared from the highly purified VLPs composed of L1 proteins of HPV types 6, 11, 16, and 18. The L1 proteins of these HPV types $(6,11,16$, and 18) are produced by separate fermentations in the recombinant Saccharomyces cerevisiae and self-assembled into VLPs. The VLPs for each type are purified and adsorbed on a aluminumcontaining adjuvant formulation-amorphous aluminum hydroxyphosphate sulfate (AAHS).

Cervarix $^{\mathrm{TM}}$ is a preparation of purified VLPs composed of L1 proteins of HPV type 16 and 18, which is formulated in AS04 adjuvant-containing aluminum hydroxide and 3-O-desacyl-4monophosphoryl lipid A (MPLA) [28]. The MPLA immunostimulant is a detoxified deriva- 
tive of the lipopolysaccharide of the bacterium Salmonella minnesota strain. The L1 proteins of these HPV types (16 and 18) are separately produced using a recombinant baculovirus expression system and the insect cell line derived from the moth-cabbage looper (Trichoplusia ni). After the expression of L1 proteins and further purification, L1 proteins are assembled separately as VLPs. It is the first vaccine licensed by the US FDA that includes MPLA as an adjuvant. The adjuvant AS04 acts as a substance incorporated into a vaccine that enhances or directs the immune response of the vaccinated individual.

The advantages that nanotechnology-based vaccines may over conventional vaccines are as follows:

1. Substitution for traditional adjuvants: Nanovaccines demonstrated immunogenicity similar to conventional vaccines without the presence of traditional adjuvant (Alum) which is found to cause irritation.

2. Extended history of safety and shelf life: Nanoparticles as vaccine delivery systems avoid the use of toxic substances such as thimerosal and formaldehyde and potentially extend the stability of vaccines on the shelf.

3. Less frequency of administration for boost: Nanovaccines can be administered for reinforcement immunization less frequently than conventional vaccines, which are usually multi-injection or multi-dose delivery systems.

\section{Perspective}

Nanotechnology may provide us a new choice for safe and effective vaccines. However, frequency of boost, refrigeration of vaccines, and routes of administration are still challenges for vaccines distribution in small areas or villages of some countries. Nanovaccines are expected to be more effective, convenient, and economic than conventional vaccines (Figure 4). We will have a great future ahead in nanovaccines if the following three aims are achieved.

1. Controlled release at specific location

Biodegradable polymers are superior to nondegradable polymers because the former may not need additional removal procedures. For reliability and reproducibility, synthetic biodegradable polymers are the best choice for antigen encapsulation in single-dose vaccine production. Biodegradable polymer microsphere such as poly-1-lactic acid (PLA) and poly lactic-co-glycolic acid (PLGA) has been used to control the time and rate of vaccine antigen release due to their safety and ability to provide long-term controlled vaccine antigen release [29]. Sustained release and pulsatile release are two possible types of vaccine antigen release with biodegradable PLGA microspheres. Sustained release, continued vaccine antigen diffusion after the initial release, mimics the administration of several small boosters. Pulsatile release, second vaccine antigen diffusion distinct from the first release, mimics the current vaccination schedule [30]. PLA and PLGA allow encapsulation within a hydrophobic core or adsorption to the hydrophilic shell, which 
can manipulate to encapsulate vaccine within the interior [31, 32]. For example, tetanus toxoid (TT) can be used to prevent tetanus which is caused by the bacterium Clostridium tetani. A pattern of constant release with a decreasing release rate after the initial burst of TT has been identified. Small-sized TT-PLA microspheres with rapid release kinetics induced an earlier release compared with larger TT-PLGA 50:50 microspheres with slow release kinetics [33]. A continuously increasing release rate after the initial burst was observed with low molecular weight TT-PLGA microspheres [34].

Nanoscale carriers can enhance the potency of vaccines to develop safer and more effective vaccine formulations. A nanoparticle-based strategy was demonstrated to safely deliver and detain intact toxins to mount a potent anti-toxin immune response. A biomimetic nanoparticle cloaked in biological membranes was used to sequester membrane-active toxins. As the event of contacting with the nanoparticles, the toxins will retrained and lose toxicity in that they are prevented from interacting with cellular targets. The resulting particles and toxin complex adopts a nanoparticulate morphology that facilitates the intracellular delivery of toxins. This technique assists to design a novel toxoid vaccine that promises more effective anti-toxin immune responses. The potent nanotoxoid formulations provide a viable anti-virulence approach to combat with microorganisms that contain membrane damaging toxins such as Staphylococcus aureus and Group A streptococcal infections [35].

2. Stable at room temperature

The common adjuvant -alum is known to cause irritation if it is used in the conventional hepatitis B vaccine. However, the use of needle-free nasal immunization with a combination of nanoemulsion and hepatitis B antigen was found to be tolerable, effective, and safe without side effects [36]. The solvent extraction or evaporation from a waterin-oil-in water $(\mathrm{W} / \mathrm{O} / \mathrm{W})$ emulsion is usually used to prepare the antigen-encapsulated nanoparticles [37]. Interestingly, refrigeration was not required for this nanoemulsion, as it was effective at $25^{\circ} \mathrm{C}$ for one month and at $40^{\circ} \mathrm{C}$ for 6 weeks, respectively [16].

To make an effort to develop nanoparticles from biodegradable and biocompatible polymers (e.g., PLA, PLGA) as vaccine delivery systems can induce both humoral and cell-mediated immunity. These biodegradable polymers may make nanovaccines have extended history of safety and proven biocompatibility at room temperature. The additional advantage is their property to control the time and rate of polymer degradation and antigen release [38].

3. Replaceable routes of administration

In addition to injection (IM, SC or ID), mouths and nostrils are two successful alternative routes for administration. The vaccine can be given by these two routes without the help of trained health workers and the equipment of sterile injection. These two methods are relatively effective, inexpensive, and convenient, compared with injection. For example, Sabin vaccine (OPV) and FluMist ${ }^{\circledR}$ are administered by oral administration and intranasal spray, respectively, in contrast to Salk vaccine (IPV) and influenza vaccine (flu shots), which are both given by injection. 
A. Sabin vaccine: An OPV is indicated for the prevention of poliomyelitis, which trivalent contains a mixture of attenuated strains of three poliovirus types. The live polioviruses vaccine produces antibodies to all types of poliovirus and triggers a local immune response in the mucous membrane of the intestines. Once infected with wild polioviruses, these antibodies protect against paralysis by restricting the viral replication inside the intestine to avoid invading to the nervous system. Later, the live attenuated vaccine virus would be excreted in

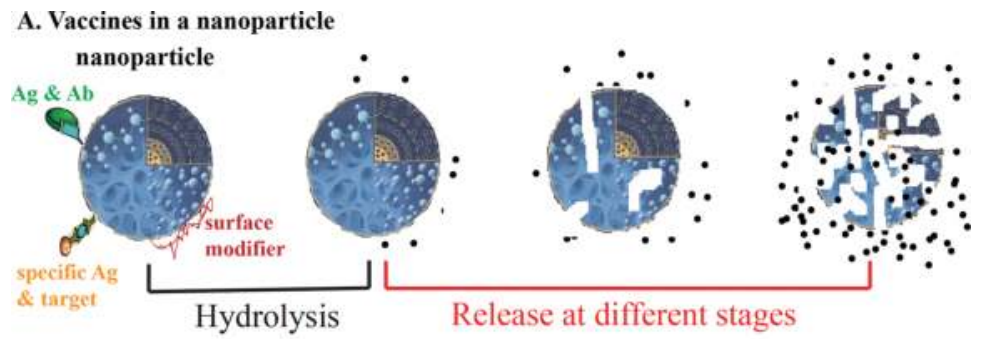

B. Vaccine boost

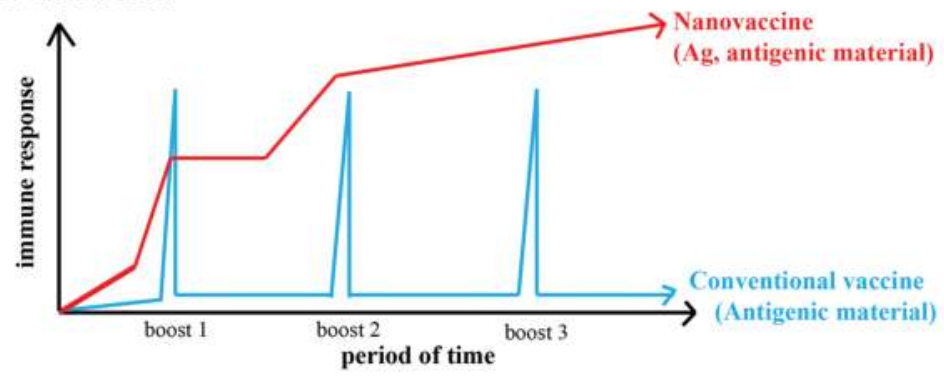

C. Interaction of nanovaccines with the immune responses

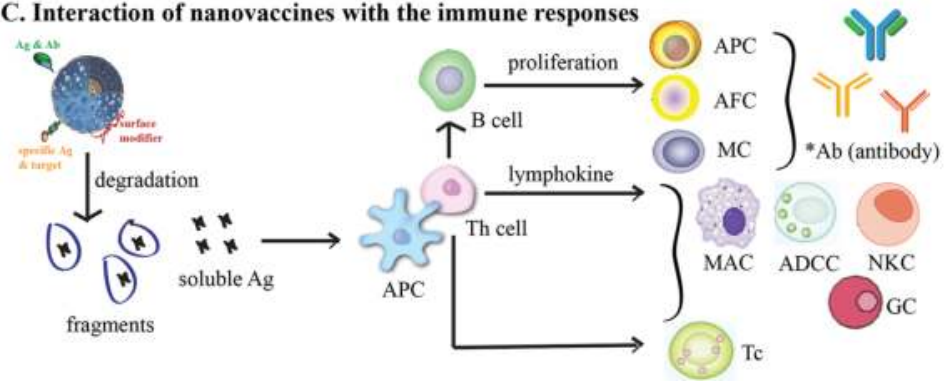

Figure 4. Nanotechnologies applied in biomedicines' vaccine (a cartoon figure for the perspective): Nanoparticles used in the vaccine production potentially overcome the disadvantages of conventional vaccines. (A) Vaccines encapsulated in nanoparticles which are polymers in solid form may be assistance in the stabilization at room temperature, elongation of storage time, exploration of alternative administration routes, and facilitation of controlled release. (B) Nanovaccines may only need to be boosted once; however, conventional vaccines usually need to be boosted many times. (C) Soluble antigens released from nanovaccines may induce both humoral immunity (B cell responses) and cell-mediated immunity (helper and cytotoxic T cell responses). ${ }^{*} \mathrm{Ab}$ (antibody): serum immunoglobulin $\mathrm{G}$ (IgG), mucosal IgG and mucosal IgA. Abbreviations: antigen (Ag); antibody (Ab); antigen presenting cell (APC); antibody forming cell (AFC); antibody-dependent cytotoxic cell (ADCC); cytotoxic T cell (Tc); granulocyte (GC); helper T cell (Th); macrophage (MAC); memory cell (MC); natural killer cell (NKC). 
the faces and spreaded to others in close contact. This means that OPV may result in the passive immunization of unvaccinated people, so that OPV can rapidly stop person-to-person transmission of wild poliovirus in areas where there is poor public health.

B. FluMist ${ }^{\circledR}$ : An intranasal spray attenuated vaccine is indicated for the prevention of influenza which quadrivalent contains four virus strains (a type A/H1N1, a type A/H3N2, and two type B strains). The live, attenuated influenza vaccine (LAIV) contains neither thimerosal nor preservatives and is sprayed into the nostrils. LAIV produces a significantly stronger immune response than inactivated vaccines, because it is delivered via the natural site of entry of the influenza virus [39-41]. In addition, this intranasal spray vaccine can prevent more $50 \%$ cases than the flu shots given by IM in younger children [42].

The skin is also an attractive organ for vaccine delivery because of its large surface area that is easily accessible for administration, and dermal delivery is almost pain-free [43, 44]. Furthermore, the skin contains many antigen presenting cells (e.g., Langerhans cells and dermal dendritic cells) and is available for the induction of immune responses [44-46]. Besides, the delivery via the skin circumvents the problem of swallowing, the first-pass effect of the liver, and the absorption and stability of vaccines in the gastrointestinal tract. However, the stratum corneum (the outermost layer of the epidermis) barrier may form significant resistance for vaccines to permeate into the body. To overcome the stratum corneum barrier, percutaneous administration such as microneedle arrays (needle-like structures with a length of less than $1 \mathrm{~mm}$ ) [43-46] is one of the most promising delivery systems.

Microparticles or nanoparticles (e.g., PLA, PLGA) have been proved to be capable of making an inactive Vibrio cholera whole-cell vaccine given by oral administration and demonstrating in vitro release in mice [47]. This is an approach using nanoparticles as an oral delivery system to improve bioavailability of vaccine and elated efficacy in therapy. Nanoparticulate material makes it possible that vaccines can be given without invasion through oral, nasal spray, and percutaneous administration such as diffusion patches, multiple puncture devices, and microneedle arrays, thus allowing painless and efficient delivery without the involvement of the professional.

\section{Conclusion}

Nanotechnology has extensively been utilized in the drug preparation and delivery for clinical applications. Nanomedicine has already been an attractive research area and the development of nanovaccines is one of the most promising enterprises. A variety of nanoparticles in vaccines have been employed as a delivery tool or immunity booster, inducing not only the highest efficacy but also the least side effects. The significance of nanovaccine development is to make it practical to have a perfect vaccination system including refrigeration-free, singledose, and painless administration. Indeed, we might have effective, safe, convenient, and cheap vaccines through nanotechnology in the near future. 


\section{Author details}

Yuan-Chuan Chen ${ }^{1}$, Hwei-Fang Cheng ${ }^{1}$, Yi-Chen Yang ${ }^{1}$ and Ming-Kung Yeh ${ }^{2,3^{*}}$

*Address all correspondence to: mkyeh2004@gmail.com

1 Food and Drug Administration, Ministry of Health and Welfare, Taipei, Taiwan

2 School of Pharmacy, National Defense Medical Center, Taipei, Taiwan

3 Ministry of Health and Welfare, Taipei, Taiwan

\section{References}

[1] Stern AM, Markel H. The history of vaccines and immunization: familiar patterns, new challenges. Health Aff. 2005; 24(3): 611-621. doi:10.1377/hlthaff.24.3.611

[2] Dunn PM. Dr. Edward Jenner (1749-1823) of Berkeley, and vaccination against smallpox. Arch. Dis. Child. Fetal Neonatal Ed. 1996; 74(1): F77-78. doi:10.1136/fn. 74.1.F77

[3] Baxby, D. Edward Jenner's Inquiry: a bicentenary analysis. Vaccine. 1999; 17(4): 301307. doi:10.1016/s0264-410x(98)00207-2

[4] Pasteur, L. Address on the germ theory. Lancet. 1881; 118(3024): 271-272. doi:10.1016/ s0140-6736(02)35739-8

[5] Stern AM, Markel H. The history of vaccines and immunization: familiar patterns, new challenges. Health Aff. 2005; 24(3): 611-621. doi:10.1377/hlthaff.24.3.611

[6] Fiore AE, Bridges CB, Cox NJ. Seasonal influenza vaccines. Curr. Top. Microbiol. Immunol. 2009; 333: 43-82. doi:10.1007/978-3-540-92165-3_3

[7] Chang Y, Brewer NT, Rinas AC, Schmitt K, Smith JS. Evaluating the impact of human papillomavirus vaccines. Vaccine. 2009; 27(32): 4355-4362. doi:10.3129/i09-126

[8] Liesegang TJ. Varicella zoster virus vaccines: effective, but concerns linger. Can. J. Ophthalmol. 2009; 44 (4): 379-384. doi:10.3129/i09-126

[9] Houghton BB, Chalasani V, Hayne D, Grimison P, Brown, CS, Patel, MI, Davis, ID, Stockler, MR. Intravesical chemotherapy plus bacille Calmette-Guérin in non-muscle invasive bladder cancer: a systematic review with meta-analysis. BJU Int. 2013; 111(6): 977-983. doi:10.1111/j.1464-410x.2012.11390.x

[10] Mosolits S, Nilsson B, Mellstedt H. Towards therapeutic vaccines for colorectal carcinoma: a review of clinical trials. Expert Rev. Vaccines. 2005; 4(3): 329-350. doi: 10.1586/14760584.4.3.329 
[11] Guidelines on the international packaging and shipping of vaccines. WHO/IVB/05.23, Printed: Dec. 2005. Available from: www.who.int/vaccines-documents/

[12] Mamo T, Poland GA. Nanovaccinology: the next generation of vaccines meets 21st century materials science and engineering. Vaccine. 2012; 30(47): 6609-6611. doi: 10.1016/j.vaccine.2012.08.023

[13] Kool M, Fierens K, Lambrecht BN. Alum adjuvant: some of the tricks of the oldest adjuvant. J. Med. Microbiol. 2012; 61(July (Pt 7)):927-934. doi:10.1099/jmm.0.038943-0

[14] Mbow ML, De GE, Valiante NM, Rappuoli R. New adjuvants for human vaccines. Curr. Opin. Immunol. 2010; 22(3): 411-416. doi:10.1016/j.coi.2010.04.004

[15] O'Hagan DT, De GE The path to a successful vaccine adjuvant - 'the long and winding road'. Drug Discov. Today. 2009; 14(11-12): 541-551. doi:10.1016/j.drudis.2009.02.009

[16] Nandedkar TD. Nanovaccines: recent developments in vaccination. J. Biosci. 2009; 34(6): 995-1003. doi:10.1007/s12038-009-0114-3

[17] Sharma S, Mukkur TK, Benson HA, Chen Y. Pharmaceutical aspects of intranasal delivery of vaccines using particulate systems. J. Pharm. Sci. 2009; 98(3): 812-893. doi: 10.1002/jps.21493

[18] Fifis T, Gamvrellis A, Crimeen-Inwin B, Pietersz GA, Li J, Mottram PL, McKenzie IFC and Plebanski M. Size-dependent Immunogenicity: therapeutic and protective properties of nanovaccines against tumors. J. Immunol. 2004; 173(5): 3148-3154. doi: 10.4049/jimmunol.173.5.3148

[19] Zhang T, Stilwell JL, Gerion D, Lianghao D, Elboudwarej O, Cooke PA, Gray JW, Alivisatos PA and Chen FF. Cellular effect of high doses of silica-coated quantum dot profiled with high throughput gene expression analysis and high content cellomics measurements. Nano Lett. 2006; 6(4): 800-808. doi:10.1021/n10603350

[20] Zhao Q, Li S, Yu H, Xia N, Modis Y. Virus-like particle-based human vaccines: quality assessment based on structural and functional properties. Trends Biotechnol. 2013; 31(11): 654-63. doi:10.1016/j.tibtech.2013.09.002

[21] Uddin MN, Kouzi SA and Hussain MD. Strategies for developing oral vaccines for human papillomavirus (HPV) induced cancer using nanoparticle mediated delivery system. Pharm. Pharm. Sci. 2015; 18(2): 220-234. Available from: http:// www.cspsCanada.org

[22] Kushnir N, Streatfield SJ, Yusibov V. Virus-like particles as a highly efficient vaccine platform: diversity of targets and production systems and advances in clinical development. Vaccine. 2012; 31(1): 58-83. doi:10.1371/j. vaccine.2012.10.083

[23] Roy P, Sutton G. New generation of African horse sickness virus vaccine based on structural and molecular studies of the virus particles. Arch. Virol. Suppl. 1998; 14:177202. doi:10.1007/978-3-7091-6823-3_17 
[24] Han MG, Cheetham S, Azevedo M, Thomas C, Saif LJ. Immune responses to bovine norovirus-like particles with various adjuvants and analysis of protection in gnotobiotic calves. Vaccine. 2006; 24(3): 317-326. doi:10.1016/j.vaccine.2005.07.071

[25] Roy P. Nature and duration of protective immunity to bluetongue virus infection. Dev. Biol. (Basel). 2003; 114:169-183. Available from: http://researchonline.lshtm.ac.uk/id/ eprint/15155

[26] Herzog C, Hartmann K, Künzi V, Kürsteiner O, Mischler R, Lazar H, Glück R. Eleven years of Inflexal V-a virosomal adjuvanted influenza vaccine. Vaccine. 2009; 27(33): 4381-4387. doi:10.1016/j.vaccine.2009.05.029

[27] Monie A, Hung CF, Roden R, Wu TC. Cervarix ${ }^{\mathrm{TM}}$ : a vaccine for the prevention of HPV 16, 18-associated cervical cancer. Biol. Targets Ther. 2008; 2(1): 107-113. Available from: http://www.ncbi.nlm.nih.gov/pmc/articles/PMC2727782/pdf/btt-2-107.pdf

[28] Kash N, Lee MA, Kollipara R, Downing C, Guidry J, Tyring SK. Safety and efficacy data on vaccines and immunization to human papillomavirus. J. Clin. Med. 2015; 4(4): 614633. doi:10.3390/jcm4040614. Review

[29] Lin CY, Lin SJ, Yang YC, Wang DY, Cheng HF, Yeh MK. Biodegradable polymeric microsphere-based vaccines and their applications in infectious diseases. Hum. Vaccines Immunother. 2015; 11(3): 650-656. doi:10.1080/21645515.2015.1009345

[30] Cleland JL. Single-administration vaccines: controlled release technology to mimic repeated immunizations. Trends Biotechnol. 1999; 17(1): 25-29. doi:10.1016/ S0167-7799(98)01272-4

[31] Yeh MK, Coombes AGA, Chen JL, Chiang CH. Japanese encephalitis virus vaccine formulations using PLA lamellar and PLG microparticles. J. Microencapsul. 2002; 19(5): 671-682. doi:10.1080/02652040210142551

[32] Huang SS, Li IH, Hong PD, Yeh MK. Development of Yersinia pestis F1 antigen-loaded microspheres vaccine against plague. Int. J. Nanomed. 2014; 9: 813-822. doi:10.2147/ IJN.S56260

[33] Alonso MJ, Cohen S, Park TG, Gupta RK, Siber GR, Langer R. Determinants of release rate of tetanus vaccine from polyester microspheres. Pharm. Res. 1993; 10(7): 945-953. doi:10.1023/A:1018942118148

[34] Alonso MJ, Gupta RK, Min C, Siber GR, Langer R. Biodegradable microspheres as controlled-release tetanus toxoid delivery systems. Vaccine. 1994; 12(4): 299-306. doi: 10.1016/0264-410X(94)90092-2

[35] Hu CM, Zhang L. Nanotoxoid vaccines. Nano Today. 2014; 9(4): 401-404. doi:10.1016/ j.nantod.2014.06.001 
[36] Makidon PE, Bielinska AV, Nigarekar SS, Janezak KW, Knowlton J, Scott AJ, Mank N, Cao Z, et al. Pre-clinical evaluation of a novel nanoemulsion-based hepatitis B mucosal vaccine. Plos One. 2008; 3(8): e2954. doi:10.1371/journal.pone.0002954

[37] Patel AR, Kulkarni SP, Nandedkar TD, Vavia PR. Evaluation of alkyl polyglucoside (based on C10 fatty alcohol) as alternative surfactant in the preparation of peptide loaded nanoparticles. J. Microencapsul. 2008; 25(8): 531-540. doi: 10.1080/02652040802075526

[38] Kersten G, Hirshberg H. Antigen delivery systems. Expert Rev. Vaccines. 2004; 3(4): 453-462. doi:10.1586/14760584.3.4.453

[39] Belshe RB, Edwards KM, Vesikari T, Black SV, Walker RE, Hultquist M, Kemble G, Connor EM; CAIV-T Comparative Efficacy Study Group. Live attenuated versus inactivated influenza vaccine in infants and young children. N. Engl. J. Med. 2007; 356(7): 685-696. doi:10.1056/NEJMoa065368

[40] Ambrose CS, Luke C, Coelingh K. Current status of live attenuated influenza vaccine in the United States for seasonal and pandemic influenza. Influenza Other Respir. Viruses. 2008; 2(6): 193-202. doi:10.1111/j.1750-2659.2008.00056.x

[41] Jefferson T, Rivetti A, Di Pietrantonj C, Demicheli V, Ferroni E. Vaccines for preventing influenza in healthy children. Cochrane Libr. 8: CD004879, 2012. doi: 10.1002/14651858.CD004879.pub4

[42] Nasal Spray Flu Vaccine in Children 2 through 8 Years Old. CDC. Center for Disease Control and Prevention, US. Retrieved on May 11, 2015. Available from: http:// www.cdc.gov/

[43] Kim YC, Park J-H, Prausnitz MR. Microneedles for drug and vaccine delivery. Adv. Drug Deliv. Rev. 2012; 64(14): 1547-1568. doi:10.1016/j.addr.2012.04.005

[44] van der Maaden K, Luttge R, Vos PJ, Bouwstra J, Kersten G, Ploemen I. Microneedlebased drug and vaccine delivery via nanoporous microneedle arrays. Drug Deliv. Transl. Res. 2015; 5(4): 397-406. doi:10.1007/s13346-015-0238-y

[45] Prausnitz MR, Langer R. Transdermal drug delivery. Nat. Biotechnol. 2008; 26(11): 1261-1268. doi:10.1038/nbt.1504

[46] Donnelly R, Douroumis D. Microneedles for drug and vaccine delivery and patient monitoring. Drug Deliv. Transl. Res. 2015; 5(4): 311-312. doi:10.1007/s13346-015-0250-2

[47] Yeh MK, Chiang CH. Inactive Vibro cholera whole-cell vaccine-loaded biodegradable microparticles: in vitro release and oral vaccination. J. Microencapsul. 2004; 21(1): 91106. doi:10.1080/02652040310001619794 
